\title{
Physical therapy in patients with disorders of consciousness: Impact on spasticity and
} muscle contracture

\author{
A. Thibaut ${ }^{\mathrm{a}, \mathrm{b}, *}$, S. Wannez ${ }^{\mathrm{a}}$, T. Deltombe ${ }^{\mathrm{c}}$, G. Martens ${ }^{\mathrm{a}}$, S. Laureys ${ }^{\mathrm{a}}$ and C. Chatelle ${ }^{\mathrm{a}, \mathrm{d}}$ \\ ${ }^{a}$ Department of Neurology, Coma Science Group, GIGA-Consciousness, University and University hospital of \\ Liege, Liege, Belgium \\ ${ }^{\mathrm{b}}$ Spaulding Neuromodulation Center, Spaulding Rehabilitation Hospital, Harvard Medical School, Boston, MA, \\ USA \\ ${ }^{\mathrm{c}}$ Department of Physical Medicine and Rehabilitation, CHU UCL Namur site Mont-Godinne, (Université \\ Catholique de Louvain), Yvoir, Belgium \\ ${ }^{\mathrm{d}}$ Laboratory for NeuroImaging of Coma and Consciousness, Massachusetts General Hospital, Boston, MA, USA
}

\begin{abstract}
.
BACKGROUND: Spasticity is a frequent complication after severe brain injury, which may prevent the rehabilitation process and worsen the patients' quality of life.

OBJECTIVES: In this study, we investigated the correlation between spasticity, muscle contracture, and the frequency of physical therapy (PT) in subacute and chronic patients with disorders of consciousness (DOC).

METHODS: 109 patients with subacute and chronic disorders of consciousness (Vegetative state/Unresponsive wakefulness syndrome - VS/UWS; minimally conscious state - MCS and patients who emerged from MCS - EMCS) were included in the study ( 39 female; mean age: $40 \pm 13.5 y$; 60 with traumatic etiology; 35 VS/UWS, 68 MCS, 6 EMCS; time since insult: $38 \pm 42$ months). The number of PT sessions (i.e., 20 to 30 minutes of conventional stretching of the four limbs) was collected based on patients' medical record and varied between 0 to 6 times per week (low PT $=0-3$ and high PT $=4-6$ sessions per week). Spasticity was measured with the Modified Ashworth Scale on every segment for both upper (UL) and lower limbs (LL). The presence of muscle contracture was assessed in every joint. We tested the relationship between spasticity and muscle contracture with the frequency of PT as well as other potential confounders such as time since injury or anti-spastic medication intake.

RESULTS: We identified a negative correlation between the frequency of PT and MAS scores as well as the presence of muscle contracture. When separating subacute ( 3 to 12 months post-insult) and chronic (>12months post-insult) patients, these negative correlations were only observed in chronic patients. A logit regression model showed that frequency of PT influenced spasticity, whereas neither time since insult nor medication had a significant impact on the presence of spasticity. On the other hand, PT, time since injury and medication seemed to be associated with the presence of muscle contracture. CONCLUSION: Our results suggest that, in subacute and chronic patients with DOC, PT could have an impact on patients' spasticity and muscles contractures. Beside PT, other factors such as time since onset and medication seem to influence the development of muscle contractures. These findings support the need for frequent PT sessions and regular re-evaluation of the overall spastic treatment for patients with DOC.
\end{abstract}

Keywords: Spasticity, hypertonicity, upper motor neuron, muscle contracture, disorders of consciousness, minimally conscious state, vegetative state/unresponsive wakefulness syndrome, non-pharmacological treatment, physical therapy

*Address for correspondence: A. Thibaut, GIGA-Consciousness, B-34, Quartier Hôpital, Avenue de l'Hôpital, 11, 4000 Liège, Belgium. Tel.: +32 3668069; E-mail: athibaut@ulg.ac.be. 


\section{Introduction}

The management of spasticity in patients who are unable to actively participate to conventional rehabilitation program is still an unresolved challenge. This motor disorder affects between 25 to $42 \%$ of patients after a stroke or a traumatic brain injury (Elovic, Simone, \& Zafonte, 2004; Urban et al., 2010) and about $88 \%$ of severely brain-injured (e.g., traumatic brain injury, anoxia or aneurysm rupture) patients with disorders of consciousness - DOC (Thibaut et al., 2014) and, despite this high prevalence, the underlying mechanisms of spasticity have been poorly investigated and are not yet fully understood. Spasticity is part of the upper motor neuron syndrome and it is usually defined as a velocitydependant increase in muscle tone (Lance, 1980). Such a disorder can induce pain, complicate cares, and alter patients' rehabilitation and quality of life (Thibaut et al., 2013). In addition, the presence of severe motor disabilities in this population may prevent a proper assessment of consciousness at the bedside (i.e. being considered unconscious, whilst actually being conscious (Cruse et al., 2011; Monti et al., 2010)), influencing medical decisions and limiting active rehabilitation (Demertzi et al., 2011). Several side-effects have also been associated with spasticity such as muscle contracture, tendon retraction and fixed equinovarus feet, among others, in different population of patients (Ada, O'Dwyer, \& O'Neill, 2006; Brainin, 2013; Svensson, Borg, \& Nilsson, 2014). These complications further increase the clinical impact of spasticity on functional recovery by impeding the patient's ability to perform activities of daily living and increasing the cost of treatment.

It is also a critical problem for caregivers, especially for mobilizations and cares. For instance, it has been shown that caregivers have to spend a greater amount of time taking care of post-stroke spastic patients as compared to patients without clinical spasticity (Ganapathy et al., 2015). Doan et al. (2012) evaluated the impact of disability in patients with post-stroke spasticity on both patients' healthrelated quality of life and caregiver burden (Doan et al., 2012). This study highlighted the relationship between patients' disability in various domains such as hygiene, dressing and pain, and a worse quality of life. Moreover, the level of patients' disability (including spasticity) and dependency in hygiene and dressing undesirably impacted caregivers' burden (Doan et al., 2012). Regarding patients with
DOC, in addition to spasticity, the lack of voluntary movements upsurges the amount of work required by caregivers leading to an increased risk of burnout (Gosseries et al., 2012). In this context, the management of spasticity may reduce the effort and time required for caregivers to perform cares and limit the risk of burnout.

Altogether, these findings highlight the importance to improve treatment options for severely braininjured non-communicative patients with DOC such as vegetative state/unresponsive wakefulness syndrome (VS/UWS - able to open their eyes but unaware of their environment and themselves (Laureys et al., 2010; The Multi-Society Task Force on PVS, 1994)) and minimally conscious state (MCS - recovery of reproducible but fluctuating signs of consciousness without functional communication (Giacino et al., 2002)). However, their limited abilities to interact with their environment limits the range of possibilities, and passive treatments such as conventional stretching (i.e., through physical therapy - PT) are usually better suited for such rehabilitation. Unfortunately, access to these treatments is not always possible in the chronic phase and is influenced by insurance policies.

The aim of the present study is to investigate the correlation between the frequency of PT per week and the severity of spasticity, as well as its correlation with muscle contracture, in both subacute and chronic patients with DOC. We hypothesise that patients who benefit from daily PT session will present less severe spasticity, as well as less associated side-effect (i.e., muscle contracture) as compared to patients receiving sporadic treatment.

\section{Methods}

\subsection{Study population}

Data were collected retrospectively from January 2011, up to June 2015. Inclusion criteria were: 1) medically stable 2) diagnosis of VS/UWS, MCS or EMCS, 3) time since insult $\geq 3$ months (subacute: 3 - 12 months and chronic: $>12$ months post-insult), and 4) age 16 and over. Exclusion criteria were: 1) documented neurological disorders previous to the acquired brain damage, and 2) presence of skin or musculoskeletal lesions (e.g., bedsores, fractures, wounds). Note that $60 \%$ of the patients $(n=65)$ were included in a previous study (Thibaut et al., 2014). These 65 participants met the same inclusion and 
exclusion criteria as the ones defined for the present study.

All patients were admitted to the University Hospital of Liège in Belgium for one week of diagnostic assessments. All patients came from their homes, nursing homes or other facilities. The study was approved by the ethical committee of the University Hospital of Liège and written informed consents were obtained from the legal representatives.

\subsection{Study design}

This retrospective cross-sectional study was based on data obtained during physical examination of patients with DOC. Spasticity and muscle contracture of the limbs (i.e., upper limbs - UL; and lower limbs - LL) were assessed by a trained physical therapist (AT).

The other outcome measures, such as the frequency of PT sessions, patients' medication and demographic characteristics, were obtained through patients' medical records.

\subsection{Outcomes measures}

\subsubsection{Muscle tone and contracture}

The tone and contracture were assessed for the elbow, the wrist, and finger flexors or extensors, as well as the hip adductors or abductors, knee, and ankle flexors or extensors, bilaterally. The tone assessment was based on the Modified Ashworth Scale (MAS), a 6-level ordinal scale with documented reliability (Bohannon \& Smith, 1987; Mehrholz et al., 2005). Higher scores indicate increasing severity of spasticity. Assessment of spasticity followed the guidelines of the scale (i.e., patients assessed in a resting position) and included passive flexion and extension of upper and lower extremity joints (shoulder, elbow, wrist, fingers, hip, knee, and ankle). The presence of spasticity was considered as MAS $\geq 1$. The median MAS score of assessable (i.e., without joint fixation preventing a valid assessment) joints of the UL (left and right shoulder, elbow, wrist, fingers) and LL (left and right hip, knee and ankle) were used for correlation analyses. The presence of muscle contracture was defined as the occurrence of a permanent shortening of a muscle or joint (Farmer \& James, 2001).

Note that the assessor was blinded from the amount of PT sessions received by each patient.

\subsubsection{Frequency of physical therapy $(P T)$}

Frequency varied between 0 to 6 times per week and consisted of 20 to 30 minutes of conventional stretching of both upper and lower extremities. To compare patients receiving "high" vs "low" rate of PT per week, we divided the frequency of PT in two groups: high PT (i.e., 4 to 6 sessions per week) and low PT (i.e., 0 to 3 sessions per week).

\subsection{Statistical analyses}

To assess the difference in demographic characteristics between the groups, we used a $t$-test for continuous variables (i.e., age and time since injury) and a Chi-square test for dichotomic variable (i.e., etiology).

The median for left and right UL and LL were used separately for analyses. We used a Spearman correlation test to assess the correlation between the frequency of PT per week (0 to 6) and MAS score (median of UL and LL - 0 to 5), for the entire group as well as for patients in subacute and chronic stage (i.e., between 3 and 12 months vs. more than 12 months post insult) separately. To compare the difference in MAS scores between patients receiving low versus high PT we used Mann Whitney test. To assess the differences in proportions (percentage of spastic versus non-spastic patients, or presence of contracture versus absence of contracture in the groups of patients receiving low versus high $\mathrm{PT}$ ) we used binomial proportion tests.

Finally, to evaluate the effect of PT, time since onset and anti-spastic medication on the presence of spasticity and muscle contracture, we performed logit regressions with 1. presence of spasticity (MAS $\geq 1$ ) and 2. muscle contracture as dependent variables and frequency of PT (low versus high), the time since onset (subacute versus chronic) and anti-spastic medication (under antispastic medication versus free of antispastic medication) intake as independent variables.

All results were considered significant at $p<0.05$.

\section{Results}

The study includes 109 patients with subacute $(n=40)$ and chronic $(n=69)$ DOC (38 female; mean age: $40 \pm 14 \mathrm{y}$; 60 with traumatic etiology; $35 \mathrm{VS} / \mathrm{UWS}, 68 \mathrm{MCS}, 6 \mathrm{EMCS}$; time since insult: $37 \pm 41$ months; subacute: 40 ; chronic: 69 ). Sixty-two patients received anti-spastic medication 
(baclofen oral $-n=47$; baclofen intratecal pomp $n=8$; Clonazepam $-n=4$; Tizanidine $-n=3$ ).

\subsection{Frequency of PT and MAS scores}

Out of the 109 patients, 36 received 0 to $3 \mathrm{PT}$ sessions per week (defined as "low PT"), and 73 received 4 to $6 \mathrm{PT}$ sessions per week (defined as "high PT"). We did not find any difference in terms of age $(\mathrm{t}=0.015 ; p=0.998)$, time since injury $(\mathrm{t}=0.566$; $p=0.572)$ or etiology $\left(\chi^{2}=0.087 ; p=0.768\right)$ between patients receiving high or low frequency of PT.

There was a significant difference in MAS scores between the two groups (low vs high PT) $(Z=-3.5622, p<0.001$ for $\mathrm{UL}$ and $Z=-3.1359$, $p=0.002$ for LL). We also identified a higher proportion of spastic patients (i.e., MAS $\geq 1$ ) among patients with low PT than among the patients with high PT $\left(94.5 \%\right.$ versus $74 \%, \chi^{2}=6.46 ; p=0.0108-$ see Table 1).

We observed a negative correlation between the frequency of PT and MAS scores for both UL and LL $(\mathrm{R}=-0.3420, p=0.0003$ and $\mathrm{R}=-0.261$, $p=0.0061$ respectively - Fig. 1$)$. When assessing the correlation in subacute and chronic patients separately, this negative correlation was observed for the chronic patients (UL: $\mathrm{R}=-0.4219, p=0.0003$ and LL: $\mathrm{R}=-0.3166, p=0.0081)$, but not for subacute patients (UL: $\mathrm{R}=-0.1951, p=0.2277$ and LL: $\mathrm{R}=-0.1590, p=0.3272$ ). The frequency of PT did not differ between subacute and chronic patients but higher MAS scores were found for UL in chronic patients as compared to subacute patients $(Z=-2.4374, p=0.015)$, while no difference was observed for LL (see Table 2).

Finally, the logit regression model was significant $\left(\chi^{2}=13.084, p=0.005\right)$, suggesting that the MAS scores could be explained by the frequency of PT $(\mathrm{OR}=0.57 ; \mathrm{T}=-2.19 ; p=0.031)$, but not by the time

Table 1

Number of patients receiving low ( 0 to 3 sessions per week) and high (4 to 6 sessions per week) rate of physical therapy (PT), and who showed signs of spasticity (MAS $\geq 1)$ or not $($ MAS $=0$ ) on the UL (upper lines) and who suffered from muscle contracture (lower lines)

\begin{tabular}{lccc}
\hline & Low PT & High PT & Total \\
\hline Spastic (MAS> $>1)$ & 34 & 54 & 88 \\
Non-spastic (MAS $=0)$ & 2 & 19 & 21 \\
Total & 36 & 73 & 109 \\
Muscle contracture & 28 & 36 & 64 \\
Absence of muscle contracture & 8 & 37 & 45 \\
Total & 36 & 73 & 109 \\
\hline
\end{tabular}

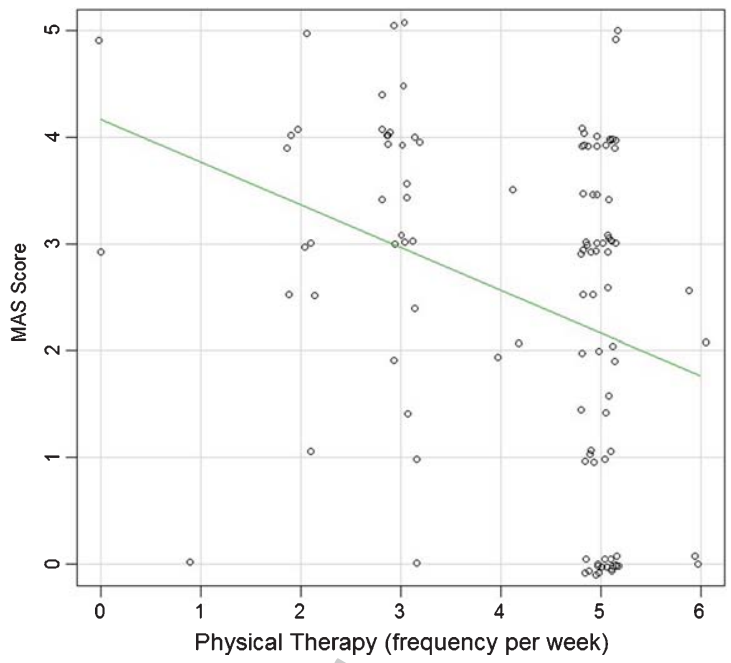

Fig. 1. Correlation between the frequency of physical therapy per week (0 to 6) and the modified Ashworth scale (MAS) scores of the upper limb (0 to 5; median of the MAS score of every segment for both right and left upper limbs). Blue circles represent each patient. Note that one circle can represent several patients with identical clinical profile. The green line represents the regression line.

Table 2

Modified Ashworth Scale (MAS) scores (median and interquartile range [IQR]) for Upper and Lower Limbs (UL \& LL) and frequency of physical therapy (PT; mean and standard deviation $[\mathrm{SD}]$ ), for patients in a subacute and chronic stage

\begin{tabular}{lccc}
\hline & $\begin{array}{c}\text { Median } \\
\text { (IQR) MAS } \\
\text { UL }\end{array}$ & $\begin{array}{c}\text { Median } \\
\text { (IQR) } \\
\text { MAS LL }\end{array}$ & $\begin{array}{c}\text { Mean (SD) } \\
\text { Frequency of PT } \\
\text { per week }\end{array}$ \\
\hline $\begin{array}{l}\text { Subacute } \\
\quad(3-12 \text { months) }\end{array}$ & $2.5(0-3)$ & $1(0-3)$ & $4.2 \pm 1.4$ \\
$\begin{array}{c}\text { Chronic } \\
\text { (>12months) }\end{array}$ & $3(1.5-4)$ & $2.5(1-4)$ & $4.2 \pm 1.2$ \\
\hline
\end{tabular}

since insult $(\mathrm{OR}=1.01 ; \mathrm{T}=1.59 ; p=0.113)$, or by medication $(\mathrm{OR}=2.29 ; \mathrm{T}=1.69 ; p=0.093)$.

\subsection{Frequency of PT and muscle contracture}

Patients with muscle contracture showed lower frequency of PT than those without contracture $(Z=2.945, p=0.003)$. In addition, muscle contracture was more often observed in patients with low PT than in patients with high PT (78\% versus $49 \%$, $\chi^{2}=8.28 ; p=0.004-$ see Table 1$)$.

A positive correlation was identified between MAS scores and the presence of muscle contracture $(\mathrm{R}=0.519 ; p<0.001)$, while a negative correlation was observed between the frequency of PT and 
the presence of muscle contracture $(\mathrm{R}=-0.2666$, $p=0.005)$. When subcategorizing patients according to their time since insult (subacute vs. chronic), this negative correlation was only significant for chronic patients $(\mathrm{R}=-0.3376, p=0.005)$, and not for subacute patients $(\mathrm{R}=-0.139, p=0.390)$.

The logit regression model was significant $\left(\chi^{2}=27.616, p<0.001\right)$, suggesting that muscle contracture could be explained by the frequency of PT $(\mathrm{OR}=0.63 ; \mathrm{T}=-2.38 ; p=0.019)$, as well as by anti-spastic medication intake $(\mathrm{OR}=5.95 ; \mathrm{T}=3.91$; $p<0.001)$, and a trend was found for the time since injury $(\mathrm{OR}=1.01 ; \mathrm{T}=1.931 ; p=0.056)$.

\section{Discussion}

In this retrospective cross-sectional study, we investigated the correlation between PT and spasticity as well as the presence of muscle contracture in patients with DOC. We reported a negative correlation between spasticity, muscle contracture and the amount of PT sessions patients received per week. More precisely, we showed that patients who received less than four sessions per week were more likely to be spastic and suffer from muscle contracture than patients receiving 4 sessions or more. This supports the hypothesis that the amount of PT influences the severity of spasticity and muscle contracture, even though no causal relationship can be drawn. If one could argue that the presence of spasticity has an effect on the frequency of PT sessions per week, it was previously shown that the amount of sessions is not determined by the patient's characteristics at baseline but rather by institution, insurance policies or other economic reasons (Thibaut et al., 2014). In addition, the severity of spasticity could not be explained by the time since insult, suggesting that PT could influences the severity of spasticity regardless of patients' chronicity.

When evaluating the impact of PT (low vs. high), time since insult and anti-spastic medication on spasticity, only PT was significantly associated with the severity of spasticity. On the other hand, for muscle contracture, PT and medication were correlated with its occurrence, while a trend was found for time since injury. Our findings are similar to what was observed in a previous study (Thibaut et al., 2014) supporting the hypothesis that patients who do not suffer from muscle contracture are not treated for it. Conversely, it could also suggest that current pharmacological treatments are not efficient enough to avoid muscle contractures development. Regarding the effect of the time since insult, the maladaptive changes related to muscle contracture are aggravated by immobilization (Gracies, 2005), which could explain why patients with DOC can develop severe muscle contractures and this symptom worsen with time.

Several systematic reviews and meta-analyses have also shown the positive effect of PT on patients' spasticity and mobility in other neurological conditions such as stroke, TBI or cerebral palsy (Autti-Rämö, Suoranta, Anttila, Malmivaara, \& Mäkelä, 2006; Borisova \& Bohannon, 2009; Hellweg \& Johannes, 2008). Hellweg and Johannes reviewed 14 studies and concluded that intensive rehabilitation programs, involving PT and occupational therapy, led to earlier functional recovery in patients with moderate to severe TBI (Hellweg \& Johannes, 2008). This was further supported by a meta-analysis including 2564 patients with moderate TBI (Turner-Stokes, Disler, Nair, \& Wade, 2005). However, these studies mainly focused on acute and subacute patients and therefore their conclusions can hardly be translated to chronic severely brain-injured patients. A recent prospective randomized clinical trial tested the effects of a wrist-hand stretching device in chronic ( $>6$ months) stroke patients suffering from spasticity (Jung et al., 2015). The authors found a significant reduction in spasticity in the treated group, highlighting the effect of stretching in reducing spasticity in a chronic population.

If our findings are supported by previous literature, they should be used with caution. Firstly, as this was cross-sectional study, we cannot state the directionality of the correlations. Longitudinal studies assessing the effects of PT needs to be done to better estimate the impact of PT on spasticity as well as its sideeffects. Secondly, we defined muscle contracture as a dichotomic variable (i.e., presence or absence of a contracture) without considering the angle, which could have given us additional information about the severity of the contracture. Thirdly, some can argue that the MAS is not the most accurate scale to assess spasticity since it does not take into account all components of hypertonia and it has not shown a good inter-rater reliability (even though, here, only one investigator assessed all patients). However, this scale seems to be most appropriate for the population we are working with (e.g., lack of voluntary movement, joint fixations, vicious positions), as it does not require active participation of the patient. Finally, since there is no definition in the literature for high and low intensity of PT, we used a threshold based 
on the guidelines for intensive (subacute setting) versus continuing (chronic setting) rehabilitation after a brain injury (i.e., $\leq 3$ versus $>3$ ). However, future studies would be needed to precise the minimal amount of sessions required to limit the occurrence of spasticity.

In conclusion, patients with DOC represent a challenging population for the treatment of spasticity due to the severe physical impairment and the lack of active collaboration limiting rehabilitation. The chronic immobilization may enhance the severity of spasticity and enhance the apparition of several sideeffects such as muscle contractures or joint fixations, leading to disuse of the limb (Kaneko, Murakami, Onari, Kurumadani, \& Kawaguchi, 2003) and affecting cares and rehabilitation. Based on our findings, the vicious circle between hypertonicity and immobilization could be partially overcome through PT, allowing regular mobilization of the patient' limb, even when no active movement is possible. Other passive treatments such as splints or motorized movement trainer (i.e. arm cycling) have also shown promising results (K Diserens et al., 2007) and could be used both in acute and chronic stage to facilitate patient's motor recovery (Karin Diserens et al., 2012), even years after the injury. From a clinical perspective, we recommend a multidisciplinary approach encompassing PT, drugs and surgery, if need be, with frequent reevaluation and adjustment in order to reduce as much as possible spasticity and its side-effects, such as muscle contractures and joint fixations, that could lead to pain and poor quality of life.

\section{Acknowledgments}

This research was supported by the Belgian Funds for Scientific Research (FRS), European Commission, EU Luminous Project, EU Human Brain Project, McDonnell Foundation, European Space Agency, Belspo, "Fondazione Europea di Ricerca Biomedica", BIAL Foundation, Wallonia-Brussels Federation Concerted Research Action, the Belgian American Educational Foundation (BAEF), the Leon Fredericq Foundation, and Wallonie Bruxelles International (WBI).

\section{Conflict of interest}

The authors have no conflict of interest to declare.

\section{References}

Ada, L., O’Dwyer, N., \& O’Neill, E. (2006). Relation between spasticity, weakness and contracture of the elbow flexors and upper limb activity after stroke: An observational study. Disabil Rehabil, 28(13-14), 891-897. http://doi.org/10.1080/09638280500535165

Autti-Rämö, I., Suoranta, J., Anttila, H., Malmivaara, A., \& Mäkelä, M. (2006). Effectiveness of upper and lower limb casting and orthoses in children with cerebral palsy: An overview of review articles. American Journal of Physical Medicine \& Rehabilitation/Association of Academic Physiatrists, 85(1), 89-103. Retrieved from http://www.ncbi.nlm. nih.gov/pubmed/16357554

Bohannon, R. W., \& Smith, M. B. (1987). Inter rater reliability of a modified Ashworth Scale of muscle spasticity. Phys Ther, 67, 206-207.

Borisova, Y., \& Bohannon, R. W. (2009). Positioning to prevent or reduce shoulder range of motion impairments after stroke: A meta-analysis. Clinical Rehabilitation, 23(8), 681686. http://doi.org/10.1177/0269215509334841

Brainin, M. (2013). Poststroke spasticity: Treating to the disability. Neurology, 80(3 Suppl 2), S1-4. Retrieved from http://www.ncbi.nlm.nih.gov/pubmed/23437441

Cruse, D., Chennu, S., Chatelle, C., Bekinschtein, T. A., Fernandez-Espejo, D., Pickard, J. D., ... Owen, A. M. (2011). Bedside detection of awareness in the vegetative state: A cohort study. Lancet, 378(9809), 2088-2094. http://doi.org/10.1016/S0140-6736(11)61224-5

Demertzi, A., Ledoux, D., Bruno, M. A., Vanhaudenhuyse, A., Gosseries, O., Soddu, A., ... Laureys, S. (2011). Attitudes towards end-of-life issues in disorders of consciousness: A European survey. J Neurol, 258(6), 1058-1065. http://doi.org/10.1007/s00415-010-5882-Z

Diserens, K., Moreira, T., Hirt, L., Faouzi, M., Grujic, J., Bieler, G., ... Michel, P. (2012). Early mobilization out of bed after ischaemic stroke reduces severe complications but not cerebral blood flow: A randomized controlled pilot trial. Clinical Rehabilitation, 26(5), 451-459. http://doi.org/10.1177/0269215511425541

Diserens, K., Perret, N., Chatelain, S., Bashir, S., Ruegg, D., Vuadens, P., \& Vingerhoets, F. (2007). The effect of repetitive arm cycling on post stroke spasticity and motor control: Repetitive arm cycling and spasticity. Journal of the Neurological Sciences, 253(1-2), 18-24. http://doi.org/10.1016/j.jns.2006.10.021

Doan, Q. V, Brashear, A., Gillard, P. J., Varon, S. F., Vandenburgh, A. M., Turkel, C. C., \& Elovic, E. P. (2012). Relationship between disability and health-related quality of life and caregiver burden in patients with upper limb poststroke spasticity. $P M R, 4(1)$, 4-10. http://doi.org/10.1016/j.pmrj.2011. 10.001

Elovic, E. P., Simone, L. K., \& Zafonte, R. (2004). Outcome assessment for spasticity management in the patient with traumatic brain injury: The state of the art. J Head Trauma Rehabil, 19(2), 155-177. Retrieved from http://www.ncbi.nlm.nih.gov/pubmed/15247825

Farmer, S. E., \& James, M. (2001). Contractures in orthopaedic and neurological conditions: A review of causes and treatment. Disability and Rehabilitation, 23(13), 549-58. Retrieved from http://www.ncbi.nlm.nih.gov/pubmed/11451189 
Ganapathy, V., Graham, G. D., DiBonaventura, M. D., Gillard, P. J., Goren, A., \& Zorowitz, R. D. (2015). Caregiver burden, productivity loss, and indirect costs associated with caring for patients with poststroke spasticity. Clinical Interventions in Aging, 10, 1793-802. http://doi.org/10.2147/CIA.S91123

Giacino, J. T., Ashwal, S., Childs, N., Cranford, R., Jennett, B., Katz, D. I., ...Zasler, N. D. (2002). The minimally conscious state: Definition and diagnostic criteria. Neurology, 58(3), 349-353. Retrieved from http://www.ncbi.nlm. nih.gov/pubmed/11839831

Gosseries, O., Demertzi, A., Ledoux, D., Bruno, M.-A., Vanhaudenhuyse, A., Thibaut, A., ... Schnakers, C. (2012). Burnout in healthcare workers managing chronic patients with disorders of consciousness. Brain Injury, 26(12), 1493-9. http://doi.org/10.3109/02699052.2012.695426

Gracies, J. M. (2005). Pathophysiology of spastic paresis. II: Emergence of muscle overactivity. Muscle Nerve, 31(5), 552-571. http://doi.org/10.1002/mus.20285

Hellweg, S., \& Johannes, S. (2008). Physiotherapy after traumatic brain injury: A systematic review of the literature. Brain Inj, 22(5), 365-373. http://doi.org/10.1080/02699050801998250

Jung, Y. J., Hong, J. H., Kwon, H. G., Song, J. C., Kim, C., Park, S., ... Jang, S. H. (2015). The effect of a wrist-hand stretching device for spasticity in chronic hemiparetic stroke patients. European Journal of Physical and Rehabilitation Medicine, 29(1), 53-59. http://doi.org/10.3233/NRE-2011-0677

Kaneko, F., Murakami, T., Onari, K., Kurumadani, H., \& Kawaguchi, K. (2003). Decreased cortical excitability during motor imagery after disuse of an upper limb in humans. Clin Neurophysiol, 114(12), 2397-2403. Retrieved from http://www.ncbi.nlm.nih.gov/pubmed/14652100

Lance, J. W. (1980). Spasticity: Disorders Motor Control. Symposium synopsis. Miami, FL: Year Book Medical Publishers: In: Feldman RG, Young RP, Koella WP eds.

Laureys, S., Celesia, G. G., Cohadon, F., Lavrijsen, J., Leon-Carrion, J., Sannita, W. G., ... Dolce, G. (2010). Unresponsive wakefulness syndrome: A new name for the vegetative state or apallic syndrome. BMC Med, 8, 68. http://doi.org/10.1186/1741-7015-8-68

Mehrholz, J., Wagner, K., Meissner, D., Grundmann, K., Zange, C., Koch, R., \& Pohl, M. (2005). Reliability of the Modified Tardieu Scale and the Modified Ashworth Scale in adult patients with severe brain injury: A comparison study. Clin Rehabil, 19(7), 751-759. Retrieved from http://www.ncbi.nlm.nih.gov/pubmed/16250194

Monti, M. M., Vanhaudenhuyse, A., Coleman, M. R., Boly, M., Pickard, J. D., Tshibanda, L., ... Laureys, S. (2010). Willful modulation of brain activity in disorders of consciousness. The New England Journal of Medicine, 362(7), 579-589. http://doi.org/10.1056/NEJMoa0905370

Svensson, J., Borg, S., \& Nilsson, P. (2014). Costs and quality of life in multiple sclerosis patients with spasticity. Acta Neurol Scand, 129(1), 13-20. http://doi.org/10.1111/ane.12139

The Multi-Society Task Force on PVS. (1994). Medical aspects of the persistent vegetative state (1). The Multi-Society Task Force on PVS. N Engl J Med, 330(21), 1499-1508. http://doi.org/10.1056/NEJM199405263302107

Thibaut, A., Chatelle, C., Wannez, S., Deltombe, T., Stender, J., Schnakers, C., ... Gosseries, O. (2014). Spasticity in disorders of consciousness: A behavioral study. Eur $J$ Phys Rehabil Med, Nov 6 [Epu. Retrieved from http://www. ncbi.nlm.nih.gov/pubmed/25375186

Thibaut, A., Chatelle, C., Ziegler, E., Bruno, M.-A., Laureys, S., \& Gosseries, O. (2013). Spasticity after stroke: Physiology, assessment and treatment. Brain Injury: [BI], 27(10), 1093105. http://doi.org/10.3109/02699052.2013.804202

Turner-Stokes, L., Disler, P. B., Nair, A., \& Wade, D. T. (2005). Multi-disciplinary rehabilitation for acquired brain injury in adults of working age. Cochrane Database of Systematic Reviews, (Issue 3). http://doi.org/10.1002/ 14651858.CD004170.pub2

Urban, P. P., Wolf, T., Uebele, M., Marx, J. J., Vogt, T., Stoeter, P., ... Wissel, J. (2010). Occurence and clinical predictors of spasticity after ischemic stroke. Stroke, 41(9), 2016-2020. 